[7] G. Köthe, Topological vector spaces $I$, Berlin 1969.

[8] C. Le $\mathrm{Page}$, Sur quelques conditions entrâ̂nant la commutativité dans les algèbres de Banach, C. R. Ácad. Sci. Paris, Sér. A 265 (1967), pp. 235-237.

[9] Gh. Mocanu, Sur quelques oritères de commutativité pour une algebre de Banach, An. Univ. București Mat.-Mec. 20 (1971), Nr. 2, pp. 127-129.

[10] V. Pták, Banach algebras with involution, Manuscripta Math. 6 (1972), pp. 245-290.

[11] V. Pták, J. Zemánek, Continuité lipschitzienne du spectre eomme fonotion d'un opérateur normal, Comment. Math. Univ. Carolinae 17 (1976), pp. 507-512.

[12] W. Rudin, Functional analysis, New York 1973. (Russian translation: Moscow 1975.)

[13] K. Srinivasacharyulu, Remarks on Banach algebras, Bull. Soc. Roy. Soi. Liège 43 (1974), pp. 523-525.

[14] C. Apostol, The spectrum and the spectral radius as functions in Banach algebras, to appear.

[15] B. A upetit, Almost commutative Banach algebras, Notices Amer. Math. Soc. 18 (1971), p. 191 .

[16] - Caractérisation spectrale des algèbres de Banach commutatives, Pacific J. Math. 63 (1976), pp. 23-35.

[17] - Propriétés spectrales des algèbres de Banach, in preparation.

[18] V. Pták, J. Z emánek, On uniform continuity of the spectral radius in Banach algebras, Manuscripta Math. 20 (1977), pp. 177-189.

[19] Z. Słodkowski, W. Wojtyński, J. Zemánek, A note on quasinilpotent elements of a Banach algebra, Bull. Acad. Polon. Sci., Sér. Sci. Math. Astronom. Phys. 25 (1977), pp. 131-134.

[20] J. Zemánek, A note on the radical of a Banach algebra, Manuscripta Math. 20 (1977), pp. 191-196.

[21] - A survey of recent results on the spectral radius in Banach algebras, Proceed ings of the Fourth Prague Symposium on General Topology and its Relations to Modern Analysis and Algebra, August 1976, to appear.

[22] - Spectral characterization of two-sided ideals in Banach algebras, to appear.

[23] - Properties of 'the spectral radius in Banach algebras, thesis, Warszawa 1977.

\section{OZECHOSLOVAK 'ACADEMY OF SCIENCES}

\section{INSTITUTE OF MATHEMATIOS}

Received February 5, $19 \%$

\section{On the Fejér-F. Riesz inequality in $L^{p}$}

by

YORAM SAGHER (Rehovot, Israel)

Abstract. Using the Lions-Peetre interpolation theory, various generalizations of a classical theorem of Fejer and F. Riesz, are proved.

Introduction. The inequality under consideration is:

$$
\left(\int_{0}^{1}|f(r, \theta)|^{p} d r\right)^{1 / p} \leqslant A_{p}\left(\int_{-\pi}^{\pi}|f(\theta)|^{p} d \theta\right)^{1 / p}, \quad 1<p \leqslant \infty
$$

where $f(r, \theta)$ is the harmonic function in $r<1$ whose boundary values are $f(\theta)$. See [3]. Fejér and Riesz proved the inequality using complex function theory so that their methods do not extend to $\boldsymbol{R}^{n}$

$N$. du Plessis was the first to generalize the theorem to $\boldsymbol{R}^{n}$. Another proof and a somewhat stronger generalization (for $n=3$ only) was given by F. R. Keogh. See [5].

Using interpolation theory we shall present a method for proving strong versions of the various theorems. The proofs are considerably simpler, and the results apply not only to the Poisson kernel, but to others as well. Even in the classical case we get, without any added difficulty, a stronger inequality:

$$
\left(\int_{0}^{1} \underset{0 \leqslant 0 \leqslant r}{\operatorname{Max}}|f(\varrho, \theta)|^{p} d r\right)^{1 / p} \leqslant A_{p}\left(\int_{-\pi}^{\pi}|f(\theta)|^{p} d \theta\right)^{1 / p} .
$$

The note is divided into two sections. In the first, we shall prove the spherical Fejér-Riesz inequalities, and in the second, the half-space versions.

We shall use freely the language and results of interpolation theory. For an outline of the theory, see for example [4], [6]. An interesting aspect of the application of interpolation theory we make here is that $L(p, q)$ spaces with $p<1$ are used in a natural way, to get results for $L^{p}$ with $1<p$. 
1. Fejér-Riesz inequalities for the sphere. $f(s) \in L^{1}\left(\Sigma_{n-1}\right)$. Luti

$$
u(x)=\gamma_{n} \int_{\Sigma_{n-1}} f(s) \frac{1-|x|^{2}}{|s-x|^{2}} d \sigma_{n-1}(s) \quad(|x|<\mathcal{1}) .
$$

We shall use the following notation: $s=\left(\zeta_{1} \ldots \zeta_{n}\right), s_{2}=\left(\zeta_{2} \ldots \zeta_{n}\right), s_{2}^{\prime}$ $=\frac{s_{2}}{\left|s_{2}\right|}, e=(1,0 \ldots 0)$.

Theorem 1. $1<p \leqslant \infty$,

$$
\begin{aligned}
& \left(\int_{0}^{1}(1-r)^{n-2} \underset{0 \leqslant \rho \leqslant r}{\operatorname{Max}}|u(\varrho e)|^{p} d r\right)^{1 / p} \\
& \quad \leqslant C_{p}\left(\int_{0}^{\pi} \sin ^{n-2} \theta\left(\left.\int_{\Sigma_{n-2}}\left|f\left(\cos \theta, \sin \theta s_{2}^{\prime}\right)\right| d \sigma_{n-2}\left(s_{2}^{\prime}\right)\right|^{p} d \theta\right)^{1 / p} .\right.
\end{aligned}
$$

Proof. $T f(r)=\operatorname{Max}\{|u(\varrho e)| 0 \leqslant \varrho \leqslant 1-r\}$. Write

$$
g(\theta)=\int_{\Sigma_{n-2}}\left|f\left(\cos \theta, \sin \theta s_{2}^{\prime}\right)\right| d \sigma_{n-2}\left(s_{2}^{\prime}\right) .
$$

We have

$$
|u(\varrho e)| \leqslant \gamma_{n} \int_{0}^{\pi} g(\theta) \frac{1-\varrho^{2}}{\left(1+\varrho^{2}-2 \varrho \cos \theta\right)^{n / 2}} \sin ^{n-2} \theta d \theta .
$$

Since

$$
\begin{aligned}
& |u(\varrho e)| \leqslant|g(\theta)|_{\infty}, \\
& |u(\varrho e)| \leqslant|g(\theta)|_{1} \frac{2 \gamma_{n}}{(1-\varrho)^{n-1}} .
\end{aligned}
$$

(We consider the function spaces on the right, on $[0, \pi]$ with measure $\sin ^{n-2} \theta d \theta$.) Therefore,

$$
T: \begin{aligned}
& S_{1}^{\infty} S_{2}^{1} \rightarrow L(\infty, \infty), \\
& S_{1}^{1} S_{2}^{1} \rightarrow L(1 /(n-1), \infty) .
\end{aligned}
$$

The spaces on the left are the mixed norm spaces. The norm of $f$ in $S_{1}^{p} S_{2}^{\alpha}$ is

$$
\left(\int_{S_{1}}\left(\int_{S_{2}}\left|f\left(s_{1}, s_{2}\right)\right|^{q} d \mu_{2}\left(s_{2}\right)\right)^{p / q} d \mu_{\mu_{1}}\left(s_{1}\right)\right)^{1 / p}
$$

where the measure spaces $\left(S_{i}, d \mu_{i}\left(s_{i}\right)\right)$ are clear from the context. In this instance: $S_{2}=\Sigma_{n-2}$, the unit sphere in $\boldsymbol{R}^{n-1}, d \mu_{2}\left(s_{2}\right)=d \sigma_{n-2}\left(s_{2}^{\prime}\right)$; $S_{1}=[-1,1]$, with the following measure: write $s_{1}=\cos \theta \cdot d \mu_{1}\left(s_{1}\right)$ $=\sin ^{n-2} \theta d \theta$. A similar mixed norm space will appear in the proof of Theorem 3.
Therefore,

$$
T: S_{1}^{p} S_{1}^{1} \rightarrow L\left(\frac{p}{(n-1)}, p\right) .
$$

Explicitly,

$$
\begin{aligned}
& \left(\int_{0}^{1}(1-r)^{n-2} \operatorname{Max}_{0 \leqslant 0<r}|u(\varrho e)|^{p} d r\right)^{1 / p} \\
& \quad \leqslant O_{p}\left(\int_{0}^{\pi} \sin ^{n-2} \theta\left(\int_{\Sigma_{n-2}}\left|f\left(\cos \theta, \sin \theta s_{2}^{\prime}\right)\right| d \sigma_{n-2}\left(s_{2}^{\prime}\right)\right)^{p} d \theta\right)^{1 / p}
\end{aligned}
$$

and the proof is complete.

This, for $n=3$ and without the Max on the left-hand side, was proved. (differently) by Keogh [5].

Note that

$$
\begin{aligned}
&\left(\int_{0}^{\pi} \sin ^{n-2} \theta\left(\int_{\Sigma_{n-2}}\left|f\left(\cos \theta, \sin \theta s_{2}^{\prime}\right)\right| d \sigma_{n-2}\left(s_{2}^{\prime}\right)\right)^{p} d \theta\right)^{1 / p} \\
& \quad \leqslant A_{n}\left(\left.\int_{\Sigma_{n-1}}|f(s)|^{p} d \sigma_{n-1}(s)\right|^{1 / p}\right.
\end{aligned}
$$

so that we have a strengthening of du Plessis' theorem.

Using an interpolation technique similar to that employed for the proof of Theorem 1, we can prove the following:

THEOREM 2. For $1<p<\infty, 0<q \leqslant \infty$, or $p=q=\infty$,

$$
\left(\int_{0}^{1}(1-r)^{(n-1) q / p-1} \operatorname{Max}_{0 \leqslant \rho \leqslant r}\left|u\left(\varrho x^{\prime}\right)\right|^{q} d r\right)^{1 / q} \leqslant C_{p, q}|f|_{\mathcal{L}(p, q)} .
$$

We shall omit the proof. This, for $q=p$ again gives the weaker consequence of Theorem 1.

When $n \geqslant 3$ one can also prove planar inequalities. Denote by $D_{n-m}$ an $n-m$ dimensional diametrical plane section in $|x|<1$. Du Plessis proved:

$$
1\left(\int_{D_{n-m}}(1-|x|)^{m-1}|u(x)|^{p} d x_{n-m}\right)^{1 / p} \leqslant O_{p}|f|_{p}, \quad 1<p<\infty .
$$

$\leqslant n-2$

We shall prove a strong version of this inequality. Denote for $1 \leqslant m$

$$
\begin{gathered}
\sin _{n}\left(\theta_{1}, \ldots, \theta_{m}\right)=\sin ^{n-2} \theta_{1} \sin ^{n-3} \theta_{2} \ldots \sin ^{n-m-1} \theta_{m}, \\
s_{1}=\left(\zeta_{1} \ldots \zeta_{m}\right), \quad s_{2}=\left(\zeta_{m+1} \ldots \zeta_{n}\right) .
\end{gathered}
$$


Theorem 3. For $1<p \leqslant \infty$, let $x=\left(0, \ldots, 0, \xi_{m+1} \ldots \xi_{n}\right)$. Then

$$
\left(\int_{0}^{1}(1-r)^{m-1} \operatorname{Max}_{0 \leqslant \varrho \leqslant r}\left(\int_{\Sigma_{n-m-1}}\left|u\left(\varrho x^{\prime}\right)\right|^{p} d \sigma_{n-m-1}\left(x^{\prime}\right)\right) d r\right)^{1 / p} \leqslant C_{p}|f|_{p} .
$$

Proof. Write

$$
T f(r)=\underset{0 \leqslant \varrho \leqslant 1-r}{\operatorname{Max}}\left(\int_{\Sigma_{n-m-1}}\left|u\left(\varrho x^{\prime}\right)\right|^{p} d \sigma_{n-m-1}\left(x^{\prime}\right)\right)^{1 / p} .
$$

We have

$$
\begin{array}{r}
u\left(\varrho x^{\prime}\right)=\gamma_{n} \int_{0}^{\pi} \ldots \int_{0}^{\pi} \sin _{n}\left(\theta_{1} \ldots \theta_{m}\right) \int_{\Sigma_{n-m-1}} f\left(s_{1}, s_{2}\right) \frac{1-\varrho^{2}}{\left(\left|s_{1}\right|^{2}+\left|s_{2}-x_{2}\right|^{2}\right)^{n / 2}} \times \\
\times d \sigma_{n-m-1}\left(s_{2}^{\prime}\right) d \theta_{1} \ldots d \theta_{m}
\end{array}
$$

where $s_{1}=\left(\cos \theta_{1}, \sin \theta_{1} \cos \theta_{2}, \ldots, \sin \theta_{1} \ldots \sin \theta_{m-1} \cos \theta_{m}\right),\left|s_{2}\right|=\sin \theta_{1} \ldots$ $\ldots \sin \theta_{m}$.

Now let $e=(0, \ldots, 0,1) . R_{x}$ is a rotation of $\Sigma_{n-m-1}$ such that $R_{x} e=x_{2}^{\prime}$ and $R_{x}$ leaves fixed all vectors perpendicular to $x_{2}^{\prime}$ and $e$. Olearly, for every fixed $s \in \Sigma_{n-m-1}$, the map of $\Sigma_{n-m-1}$ onto itself $x \rightarrow R_{x} s$ is measure preserving so that for any $\varphi$

$$
\int_{\Sigma_{n-m-1}} \varphi\left(R_{x} s\right) d \sigma_{n-m-1}\left(x^{\prime}\right)=\int_{\Sigma_{n-m-1}} \varphi(s) d \sigma_{n-m-1}(s) .
$$

Let $t_{2}$ be defined by $s_{2}=R_{x} t_{2}$. We have

$$
\begin{aligned}
u\left(\varrho x^{\prime}\right)=\gamma_{n} \int_{0}^{\pi} \ldots \int_{0}^{\pi} \sin _{n}\left(\theta_{1}, \ldots, \theta_{m}\right) \int_{\Sigma_{n-m-1}} f\left(s_{1}, R_{x} t_{2}\right) \times \\
\quad \times \frac{1-\varrho^{2}}{\left(1+\varrho^{2}-2 \varrho\left(t_{2}, e\right)\right)^{n / 2}} d \sigma_{n-m-1}\left(t_{2}^{\prime}\right) d \theta_{1} \ldots d \theta_{m} .
\end{aligned}
$$

Using Minkowski's inequality,

$$
\begin{aligned}
& \left(\int_{\Sigma_{n-m-1}}\left|u\left(\varrho x^{\prime}\right)\right|^{p} d \sigma_{n-m-1}\left(x^{\prime}\right)\right)^{1 / p} \\
& \leqslant \gamma_{n} \int_{0}^{\pi} \ldots \int_{0}^{\pi} \sin _{n}\left(\theta_{1} \ldots \theta_{m}\right) \int_{\Sigma_{n-m-1}} \frac{1-\varrho^{2}}{\left(1+\varrho^{2}-2 \varrho\left(t_{2}, e\right)\right)^{n / 2}} \times \\
& \times\left(\int_{\Sigma_{n-m-1}}\left|f\left(s_{1}, R_{x} t_{2}\right)\right|^{p} d \sigma_{n-m-1}\left(x^{\prime}\right)\right)^{1 / n} d \sigma_{n-m-1}\left(t_{2}^{\prime}\right) d \theta_{1} \ldots d \theta_{m} . \\
& \left(\int_{\Sigma_{n \rightarrow m-1}}\left|f\left(s_{1}, R_{x} t_{2}\right)\right|^{p} d \sigma_{n-m-1}\left(x^{\prime}\right)\right)^{1 / p}=\left(\int_{\Sigma_{n-m-1}}\left|f\left(s_{1}, u_{2}\right)\right|^{p} d \sigma_{n-m-1}\left(u_{2}^{\prime}\right)\right)^{1 / p} \\
& =\left|f\left(s_{1}, s_{2}\right)\right|_{S_{2}^{p}} .
\end{aligned}
$$

Thus,

$$
\begin{aligned}
& \left(\int_{\Sigma_{n-m-1}}\left|u\left(\varrho x^{\prime}\right)\right|^{p} d \sigma_{n-m-1}\left(x^{\prime}\right)\right)^{1 ! p} \\
& \leqslant \gamma_{n} \int_{0}^{\pi} \ldots \int_{0}^{\pi} \sin _{n}\left(\theta_{1} \ldots \theta_{m}\right) \int_{\Sigma_{n-m-1}} \frac{1-\varrho^{2}}{\left(1+\varrho^{2}-2 \varrho\left(t_{2}, e\right)\right)^{n / 2}} \times \\
& \quad \times\left|f\left(s_{1}, s_{2}\right)\right|_{S_{2}^{p}} d \sigma_{n-m-1}\left(t_{2}^{\prime}\right) d \theta_{1} \ldots d \theta_{m}
\end{aligned}
$$

and we have

$$
T f(r) \leqslant|f|_{S_{1}^{\infty}\left(S_{2}^{p}\right)}
$$

On the other hand, we have:

$$
\begin{aligned}
& \int_{\Sigma_{n-m-1}} \frac{1-\varrho^{2}}{\left(1+\varrho^{2}-2 \varrho\left(t_{2}, e\right)\right)^{n / 2}} d \sigma_{n-m-1}\left(t_{2}^{\prime}\right) \\
\leqslant & \frac{1}{(1-\varrho)^{m}} \cdot \int_{\Sigma_{n-m-1}} \frac{1-\varrho^{2}}{\left(1+\varrho^{2}-2 \varrho\left(t_{2}, e\right)\right)^{(n-m) / 2}} d \sigma_{n-m-1}^{\circ}\left(t_{2}^{\prime}\right) \leqslant \frac{1}{\gamma_{n-m}} \frac{1}{(1-\varrho)^{m}} .
\end{aligned}
$$

Therefore,

$$
T f(r) \leqslant \frac{\gamma_{n}}{\gamma_{n-m}}|f|_{S_{1}^{1} S_{2}^{p}} r^{-m},
$$

i.e.,

$$
T: S_{1}^{1} S_{2}^{p} \rightarrow L(1 / m, \infty),
$$$$
T: S_{1}^{\infty} S_{2}^{p} \rightarrow L(\infty, \infty) \text {. }
$$

Interpolating, we get

$$
\text { Explicitly, }
$$$$
T: S_{1}^{p} S_{2}^{p} \rightarrow L(p / m, p)
$$

$$
\begin{aligned}
& \left(\int_{0}^{1}(1-r)^{m-1} \operatorname{Max}_{0 \leqslant \rho \leqslant r} \int_{\Sigma_{n-m-1}}\left|u\left(\varrho o^{\prime}\right)\right|^{p} d \sigma_{n-m-1}\left(x^{\prime}\right) d r\right)^{1 / p} \\
\leqslant & C_{p}\left(\int_{0}^{\pi} \ldots \int_{0}^{\pi} \sin _{n}\left(\theta_{1} \ldots \theta_{m}\right) \int_{\Sigma_{n-m-1}}\left|f\left(s_{1}, s_{2}\right)\right|^{p} d \sigma_{n-m-1}\left(s_{2}^{\prime}\right) d \theta_{1} \ldots d \theta_{m}\right)^{1 / p} \\
= & C_{p}|f|_{L^{p}\left(\Sigma_{n-1}\right)} .
\end{aligned}
$$

Replacing the left-hand side of the inequality by the smaller

$$
\begin{aligned}
\left(\int_{0}^{1}(1-r)^{m-1} \int_{\Sigma_{n-m-1}}\left|u\left(r x^{\prime}\right)\right|^{p} d \sigma_{n-m-1}\left(x^{\prime}\right) r^{n-m-1} d r\right)^{1 / p} & \\
& =\left(\int_{D_{n-m}}(1-|x|)^{m-1}|u(x)|^{p} d x_{n-m}\right)^{1 / p}
\end{aligned}
$$

we get du Plessis' theorem. 
The constant $C_{p}$ both in Theorem 1 and in Theorem 3 satisfies $C_{p}$ $\leqslant \frac{A}{p-1}$ as $p \rightarrow 1^{+}$and $C_{p} \leqslant A p$ as $p \rightarrow \infty$. See, e.g. [4], Theorem 3.2 . (The theorem is stated there for linear operators. However, the proof goes through for quasi-linear and certainly for our operators.) This is important for it implies Orlicz space results:

THEOREM 4. Suppose $0 \leqslant T f, T(f+g) \leqslant T f+T g, T(\lambda f)=|\lambda| T f$. Suppose also that

$$
|T f|_{L^{p}\left(X_{1}, \mu_{1}\right)} \leqslant \frac{A}{(p-1)^{\delta}}|f|_{L^{p}\left(X_{0}, \mu_{0}\right)}
$$

where $\mu_{i}\left(X_{i}\right)<\infty$, for all $1<p<p_{0}$. Then

$$
\int_{x_{1}} T f d \mu_{1} \leqslant A \int_{x_{0}}|f|\left(\log ^{+}|f|\right)^{\delta} d \mu_{0}+B
$$

The proof of Theorem XII, 4.41 of [7] goes through with minor changes here. In [7] the operator is assumed to be linear, but on the other hand there is of course no assumption of positivity. Theorem 4 applies in other contexts as well, most importantly to the Hardy-Littlewood maximal function. With this observation we get immediately:

\section{THEOREM 5.}

$$
\int_{0}^{1}(1-r)^{n-2} \underset{0 \leqslant \varrho \leqslant r}{\operatorname{Max}}|u(\varrho e)| d r \leqslant A \int_{0}^{\pi} \sin ^{n-2} \theta g(\theta) \log ^{+} g(\theta) d \theta+B,
$$

where

$$
g(\theta)=\int_{\Sigma_{n-2}}\left|f\left(\cos \theta, \sin \theta s_{2}^{\prime}\right)\right| d \sigma_{n-2}\left(s_{2}^{\prime}\right) .
$$

Applying Jensen's inequality to Theorem 5 or else applying Theorem 4 to a weak form of Theorem 1, we get the weaker

THEOREM 6.

$$
\int_{0}^{1}(1-r)^{n-2} \underset{0 \leqslant \ell \leqslant r}{\operatorname{Max}}\left|u\left(\varrho x^{\prime}\right)\right| d r \leqslant A \int_{\Sigma_{n \rightarrow 1}}|f(s)| \log ^{+}|f(s)| d \sigma_{n-1}(s)+B .
$$

This is a strong form of a theorem of P. S. Bullen (see [2]).

Next, considering the operator

we have

$$
T: f \rightarrow u,
$$

$$
|T f|_{L^{P}\left(D_{n-m},(1-|x|)^{m-1} d x_{n-m}\right)} \leqslant \frac{A}{p-1}|f|_{L^{p}\left(\Sigma_{n-1}\right)}
$$

so that we get du Plessis' theorem:
THEOREM 7.

$\int_{D_{n-m}}(1-|x|)^{m-1}|u(x)| d x_{n-m} \leqslant A \int_{\Sigma_{n-1}}|f(s)| \log ^{+}|f(s)| d \sigma_{n-1}(s)+B$.

Finally, applying the first part of Theorem XII 4.41 of [7]. (Here we need no changes. Although the theorem there is stated for linear operators this part goes through verbatim for sublinear ones.), we have

ThEorim 8. There exist $0<\lambda, K$ such that

$$
\begin{gathered}
\int_{0}^{1}(1-r)^{n-2} \exp (\lambda \underset{0 \leqslant 0 \leqslant r}{\operatorname{Max}}|u(\varrho e)|) d r \leqslant K, \\
\int_{D_{n-m}}(1-|x|)^{m} \exp |\lambda u(x)| d x_{n-m} \leqslant K
\end{gathered}
$$

if $|f(s)| \leqslant 1$.

2. Fejér-Riesz inequalities for the half space.

THEOREM 1. $\eta(x) \in L^{1} \cap L^{\infty}$. Define for $t>0$

$$
T_{\eta} f(t)=\sup _{t \leqslant u}\left|\frac{1}{u^{n}} \int_{\boldsymbol{R}^{n}} f(x) \eta\left(\frac{x}{u}\right) d x\right| .
$$

Then, for $1<p<\infty, 0<q \leqslant \infty$ and for $p=q=\infty$,

Proof.

$$
\left|T_{\eta} f\right|_{L(p, q)} \leqslant C_{p, q}|f|_{L(p, q)}
$$

$$
\left|\frac{1}{u^{n}} \int_{\boldsymbol{R}^{n}} f(x) \eta\left(\frac{x}{u}\right) d x\right| \leqslant\left\{\begin{array}{l}
|\eta|_{x}|f|_{\infty} \\
\frac{1}{u^{n}}|\eta|_{\infty}|f|_{1}
\end{array} .\right.
$$

Therefore,

$$
T: \begin{aligned}
& L(1,1) \rightarrow L(1 / n, \infty) \\
& L(\infty, \infty) \rightarrow L(\infty, \infty)
\end{aligned}
$$

so that

$$
T: L(p, q) \rightarrow L(p / n, q) \text {. }
$$

Explicitly,

$$
\left(\int_{0}^{\infty} t^{n \alpha / p-1} \sup _{t \leqslant u}\left|\frac{1}{u^{n}} \int_{\boldsymbol{n}^{n}} f(x) \eta(x / u) d x\right|^{\alpha} d t\right)^{1 / q} \leqslant O_{p, q}|f|_{p, q} .
$$

The weaker result one gets by dropping the sup on the left-hand side of the last inequality, specializes for $q=p$ and

$$
\eta(x)=\alpha_{n} \frac{1}{\left(1+|x|^{2}\right)^{(n+1) / 2}}
$$


to du Plessis' result

$$
\left(\int_{0}^{\infty}\left|f\left(y_{1}, \ldots, y_{n}, t\right)\right|^{p} t^{n-1} d t\right)^{1 \mid p} \leqslant C_{p}\left(\int_{\boldsymbol{R}^{n}}|f(x)|^{p} d x\right)^{1 / p}
$$

where $f\left(x_{1}, \ldots, x_{n}, t\right)$ is the function harmonic in $\boldsymbol{R}^{n} \times T^{+}$whose boundary values are $f(x)$. We can, however, prove a still stronger result under an additional requirement on $\eta(x)$ - satisfied by Poisson's kernel:

THeorem 2. $\eta(x) \in L^{1} \cap L^{\infty}$, and $\eta(x)$ is radial. $T_{\eta} f(t)$ is defined as before. Then, for $1<p \leqslant \infty$,

$$
\left(\int_{0}^{\infty} t^{n-1}\left(T_{\eta} f(t)\right)^{p} d t\right)^{1 / p} \leqslant C_{p}\left(\int_{0}^{\infty} \varrho^{n-1}\left(\int_{\Sigma_{n-1}}\left|f\left(\varrho x^{\prime}\right)\right| d \sigma_{n-1}\left(x^{\prime}\right)\right)^{p} d \varrho\right)^{1 / p} .
$$

\section{Proof.}

$$
\begin{aligned}
\frac{1}{u^{n}} \int_{\boldsymbol{R}^{n}} f(x) \eta\left(\frac{x}{u}\right) d x & =\frac{1}{u^{n}} \int_{0}^{\infty} \varrho^{n-1}\left(\int_{\Sigma_{n-1}} f\left(\varrho x^{\prime}\right) \eta\left(\frac{\varrho x^{\prime}}{u}\right) d \sigma_{n-1}\left(x^{\prime}\right)\right) d \varrho \\
& =\frac{1}{u^{n}} \int_{0}^{\infty} \varrho^{n-1} \eta\left(\frac{\varrho e}{u}\right) \int_{\Sigma_{n-1}} f\left(\varrho x^{\prime}\right) d \sigma_{n-1}\left(x^{\prime}\right) d \varrho,
\end{aligned}
$$

where $e=(1,0, \ldots, 0)$.

Write

$$
|f|_{L^{p} L^{1}}=\left(\int_{0}^{\infty} \varrho^{n-1}\left(\int_{\Sigma_{n-1}}\left|f\left(\varrho x^{\prime}\right)\right| d \sigma_{n-1}\left(x^{\prime}\right)\right)^{p} d \varrho\right)^{1 / \infty}
$$

We have

Therefore:

$$
\begin{aligned}
& \left|T_{\eta} f(t)\right| \leqslant C|f|_{L^{\infty} L^{1}}|\eta|_{L^{1}}, \\
& \left|T_{\eta} f(t)\right| \leqslant|f|_{L^{1} L^{1}}|\eta|_{L^{\infty}} t^{-n} .
\end{aligned}
$$

so that

$$
T_{\eta}: \begin{aligned}
& L^{\infty} L^{1} \rightarrow L(\infty, \infty) \\
& L^{1} L^{1} \rightarrow L(1 / n, \infty)
\end{aligned}
$$

i.e.,

$$
T_{\eta}: L^{p}\left(L^{1}\right) \rightarrow L(p / n, p)
$$

$$
\left(\int_{0}^{\infty} t^{n-1}\left(T_{\eta} f(t)\right)^{p} d t\right)^{1 / p} \leqslant C_{p}\left(\int_{0}^{\infty} \varrho^{n-1}\left(\int_{\Sigma}\left|f\left(\varrho x^{\prime}\right)\right| d \sigma_{n-1}\left(x^{\prime}\right)\right)^{p} d \varrho\right)^{1 / p} .
$$

The theorem is proved.

\section{Since}

$$
\begin{aligned}
& \left(\int_{0}^{\infty} \varrho^{n-1}\left(\int_{\Sigma_{n-1}}\left|f\left(\varrho x^{\prime}\right)\right| d \sigma_{n-1}\left(x^{\prime}\right)\right)^{p} d \varrho\right)^{1 / p} \\
& \quad \leqslant C_{p, n}\left(\int_{0}^{\infty} \varrho^{n-1} \int_{\Sigma_{n-1}}\left|f\left(\varrho x^{\prime}\right)\right|^{p} d \sigma_{n-1}\left(x^{\prime}\right) d \varrho\right)^{1 / p}=C_{p, n}|f|_{p} .
\end{aligned}
$$

We have, for radial $\eta$, a strong version of the case $q=p$ of Theorem 1 . The inequality for harmonic functions is

$$
\begin{aligned}
& \left(\int_{0}^{\infty} t^{n-1} \sup _{t \leqslant u}\left|f\left(y_{1}, \ldots, y_{n}, u\right)\right|^{p} d t\right)^{1 / p} \\
& \quad \leqslant\left(\int_{0}^{\infty} \varrho^{n-1}\left(\int_{\Sigma_{n-1}}\left|f\left(y-\varrho x^{\prime}\right)\right| d \sigma_{n-1}\left(x^{\prime}\right)\right)^{p} d \varrho\right)^{1 / p} .
\end{aligned}
$$

Theorems 1 and 2 correspond to radial inequalities. We next prove the planar inequalities. We again use the mixed norm notation.

THEOREM 3. Write $y_{1}=\left(\zeta_{1} \ldots \zeta_{m}\right), \quad y_{2}=\left(\zeta_{m+1} \ldots \zeta_{n}\right), d y_{1}=d \zeta_{1} \ldots$ ...d $\zeta_{m}$, etc. Assume $\eta(x)=\eta\left(x_{1}, x_{2}\right) \in X_{2}^{1}$ for each $x_{1} \in \boldsymbol{R}^{m}$, and that

$$
K\left(x_{1}\right)=\int_{\boldsymbol{R}^{n-m}}\left|\eta\left(x_{1}, x_{2}\right)\right| d x_{2} \in X_{1}^{1} \cap X_{1}^{\infty} .
$$

\section{Write}

$$
T f(t)=\sup _{t \leqslant u}\left|\frac{1}{u^{n}} \int_{\boldsymbol{R}^{n}} \eta\left(-\frac{x_{1}}{u}, \frac{y_{2}-x_{2}}{u}\right) f(x) d x\right|_{Y_{2}^{p}} .
$$

Then, for $1<r<\infty, 0<q \leqslant \infty$ and for $r=q=\infty$,

Proof.

$$
\left(\int_{0}^{\infty} t^{m^{q} / r-1}(T f(t))^{q} d t\right)^{1 / q} \leqslant C_{p, q, r}|f|_{X_{1}^{r, q} X_{2}^{p}}
$$

$$
\begin{aligned}
\mid \frac{1}{u^{n}} \int_{\boldsymbol{R}^{n}} \eta & \left.\left(-\frac{x_{1}}{u}, \frac{y_{2}-x_{2}}{u}\right) f(x) d x\right|_{Y_{2}^{p}} \\
& \leqslant \frac{1}{u^{n}} \int_{\boldsymbol{R}^{n}}\left|\eta\left(\frac{x}{u}\right)\right|\left(\int_{\boldsymbol{R}^{n-m}}\left|f\left(-x_{1}, y_{2}-x_{2}\right)\right|^{p} d y_{2}\right)^{1 / p} d x \\
& =\frac{1}{u^{m}} \int_{\boldsymbol{R}^{m}} d x_{1}\left(\int_{\boldsymbol{R}^{n-m}}\left|n\left(\frac{x_{1}}{u}, x_{2}\right)\right| d x_{2}\right)\left(\int_{\boldsymbol{R}^{n-m}}\left|f\left(-x_{1}, y_{2}\right)\right|^{p} d y_{2}\right)^{1 / p} \\
& =\frac{1}{u^{m}} \int_{\boldsymbol{R}^{m}} K\left(\frac{x_{1}}{u}\right)\left(\int_{\boldsymbol{R}^{n-m}}\left|f\left(-x_{1}, y_{2}\right)\right|^{p} d y_{2}\right)^{1 / p} d x_{1} .
\end{aligned}
$$


Therefore

$$
T: \begin{aligned}
& X_{1}^{\infty} X_{2}^{p} \rightarrow L(\infty, \infty) \\
& X_{1}^{1} X_{2}^{p} \rightarrow L(1 / m, \infty)
\end{aligned}
$$

Interpolating,

$$
T: X_{1}^{r, q} X_{2}^{p} \rightarrow L(r / m, q) .
$$

The theorem is proved.

Again, taking $r=q=p$ and $\eta=$ Poisson kernel, we get

$$
\left(\int_{0}^{\infty}\left(\sup _{t \leqslant u} \int_{\boldsymbol{R}^{n-m}}\left|f\left(y_{1} \ldots y_{n}, u\right)\right|^{p} d y_{m+1} \ldots d y_{n}\right) t^{m-1} d t\right)^{1 / p} \leqslant O_{p}|f|_{L^{p}\left(\boldsymbol{R}^{n}\right)} .
$$

We can strengthen the last conclusion somewhat by applying the following theorem (the notation is the same as in Theorem 3 ).

- THeOREM 4. Under the assumptions of Theorem 3 , if $K\left(x_{1}\right)$ is also radial

$$
\begin{aligned}
& \left(\int_{0}^{\infty} t^{m-1}(T f(t))^{p} d t\right)^{1 / p} \\
& \quad \leqslant C_{p}\left(\int_{0}^{\infty} \varrho^{m-1} \int_{\boldsymbol{R}^{n-m}}\left(\int_{\Sigma_{m-1}}\left|f\left(\varrho y_{1}^{\prime}, y_{2}\right)\right| d \sigma_{m-1}\left(y_{1}^{\prime}\right)\right)^{p} d y_{2} d \varrho\right)^{1 / p} .
\end{aligned}
$$

We omit the proof.

\section{References}

[1] N. du Plessis, Half space analogues of the Fejer-Riesz theorem, J. London Math. Soc. 30 (1955), pp. 296-301.

[2] - Spherical Fejér-Riesz theorems, ibid. 31 (1956), 386-391.

[3] L. Fejér and F. Riesz, Über einige funotionentheoretische Ungleiohungen, Math. Zeitschrift 11 (1921), pp. 305-314.

[4] T. Holmstedt, Interpolation of quasi-normed spaces, Math. Soand. 26 (1970), pp. 177-199.

[5] F. R. Keogh, On a theorem of Fejér and Riesz, Proc. Amer. Math. Soc. 20 (1969), pp. $45-50$.

[6] J. Peetre and G. Sparr, Interpolation of normed abelian groups, Annali di Mat. Pura ed App. (IV) 42 (1972), pp. 217-262.

[7] A. Zygmund, Trigonometrio series, Cambridgo Univ. Press, Cambridge 1959

\section{DEPARTMENT OF THEORETTOAT MATHEMATIOS}

DHe

REHOVOT, ISRAET

\section{Weighted norm inequalities for parabolic fractional integrals}

by

ROBERTO A. MACIAS and CARLOS SEGOVIA (São Paulo, Brazil)

Abstract. Norm inequalities are obtained for parabolic fractional integrals of distributions whose maximal functions belong to $L^{p}\left(\boldsymbol{R}_{n}, \omega(x) d x\right)$, where $0<p<\infty$ and $\omega(x)$ is a weight satisfying an $A^{p}$-type condition and an anti-Hölder condition.

§ 1. Introduction. In this paper we obtain weighted norm inequalities for parabolic fractional integrals of distributions. The explicit definition of this fractional integrals and their existence are given in Theorem 2 . The norm inequalities are stated in Theorem 5. The unweighted case has already been considered by A. P. Calderón and A. Torchinsky (see [1], [2] and [1.0]). For the classic case of harmonic functions and $p>1$ weighted norm inequalities were obtained by B. Muckenhoupt and R. L. Wheeden in [9].

The basis of our method is a generalization of a result due to $\mathrm{L}$. Carleson and extended by P. L. Duren (see [3] and [5]) and the result stated in Theorem 4. Theorem 5 is obtained from Theorem 4 by applying some techniques developed by L. I. Hedberg in [7] and G. V. Welland in [11] for the weighted case. A similar method but technically much simpler was already used in. [8] in order to extend the results of B. Muckenhoupt and R. L. Wheeden in [9].

\$2. Definitions and notations. We shall consider an $n \times n$ real matrix $P$, satisfying $(P x, x) \geqslant(x, x)$ for every $x \in \boldsymbol{R}_{n}$, where $(y, x)$ indicates the ordinary inner product in the $n$-dimensional Euclidean space $\boldsymbol{n}_{n}$. The transpose of $P$ with respect to this inner product will be denoted by $P^{*} . P$ defines the continuous group of transformations $\left\{t^{P}\right\}_{t>0}$, where $t^{P}=e^{\ln t \cdot t^{2}}$. For $x \in \boldsymbol{R}_{n}, x \neq 0$, the function $\varrho(x)$ is defined as the unique value of $t$ such that $\left|t^{-P} x\right|=1$, where $|x|$ designates the norm of $x$ in $\boldsymbol{R}_{n}$, and $\varrho(0)=0$. The function $\varrho(x)$ satisfies $\varrho\left(t^{P} x\right)=t \varrho(x)$ and $\varrho(x+y)$ $\leqslant \varrho(x)+\varrho(y)$, thus it defines a translation invariant metric $\varrho(x-y)$. likewise, since $\left(P^{*} x, x\right)=\left(x, P_{x}\right) \geqslant(x, x)$, we can associate to $P^{*}$ a function $\varrho^{*}(x)$. Wo shall say that a function $\Omega(x)$ is $\varrho$-homogeneous of degree $m$ 